Nouvelles perspectives en sciences sociales

Revue internationale de systémique complexe et d'études relationnelles

\title{
Une approche dialectique de la ville en train de se faire : confrontation des représentations des concepteurs et des habitants de l'écoquartier de Bottière-Chénaie
}

\section{Matthieu Adam et Georges-Henry Laffont}

Volume 10, numéro 1, novembre 2014

Sur le thème du territoire

URI : https://id.erudit.org/iderudit/1028440ar

DOI : https://doi.org/10.7202/1028440ar

Aller au sommaire du numéro

Éditeur(s)

Prise de parole

ISSN

1712-8307 (imprimé)

1918-7475 (numérique)

Découvrir la revue

Citer cet article

Adam, M. \& Laffont, G.-H. (2014). Une approche dialectique de la ville en train de se faire : confrontation des représentations des concepteurs et des habitants de l'écoquartier de Bottière-Chénaie. Nouvelles perspectives en sciences sociales, 10(1), 193-235. https://doi.org/10.7202/1028440ar
Résumé de l'article

Cet article entend participer à une démarche de caractérisation de la production de la ville en ce début de XXI ${ }^{\mathrm{e}}$ siècle. Pour ce faire, il s'appuie sur une modélisation du projet urbain en phase de réalisation comme un dispositif de médiation entre ces les représentations de ses concepteurs et ses récepteurs (les habitants). Nous souhaitons comprendre comment elles se construisent et s'influencent mutuellement afin d'appréhender pourquoi elles ne se rejoignent pas.

Cette approche dialectique met en exergue la pluralité de sens qui peuvent être accordés aux propriétés matérielles et symboliques d'un territoire. Par l'analyse des représentations des concepteurs et des habitants de l'écoquartier de Bottière-Chénaie à Nantes, nous montrerons les relations qui existent entre celles-ci et la nécessité de dépasser la vision simplificatrice d'un processus linéaire du projet - conception, réalisation, réception - et qu'il faut intégrer dans le champ de la recherche urbaine des modèles intégrant les influences des effets sur les causes. 


\title{
Une approche dialectique de la ville en train de se faire : confrontation des représentations des concepteurs et des habitants de l'écoquartier de Bottière-Chénaie
}

\author{
Matthieu Adam et Georges-Henry Laffont \\ UMR CNRS CITERES équipe IPAPE, \\ Université François-Rabelais de Tours
}

'histoire des villes se constitue moins par enchaînement de
ruptures successives que par le renouvellement progressif des configurations discursives et matérielles de sa production et des territoires qu'elle engendre ${ }^{1}$. Les territoires de la ville contemporaine sont alors les fruits de l'héritage des modes de production précédents et des renouvellements récents dont ils portent alors les valeurs. En l'occurrence, l'urbanisme contemporain se caractérise essentiellement, en ce qui concerne son mode de production, par l'avènement généralisé de la gestion par projet et, pour ce qui est de son fondement idéologique, par son basculement dans l'aire du durable.

Écoquartiers, quartiers durables, etc. peuvent être considérés comme les réalisations emblématiques de la production contemporaine de l'urbain. Leur étude permet de répondre à trois questions : comment se caractérise la production de la ville en ce

Françoise Choay, La règle et le modèle : sur la théorie de l'architecture et de l'urbanisme, Paris, Seuil, 1996 [1980]. 
début de $\mathrm{XXI}^{\text {e }}$ siècle? De quelles manières les quartiers durables sont-ils conçus par ceux qui en ont la charge et par ceux qui les habitent? Quels décalages sont identifiables?

Influencés notamment par l'étude des représentations en psychologie sociale ${ }^{2}$ et environnementale ${ }^{3}$ ainsi que par la géographie $^{4}$, nos travaux s'inscrivent - en première approche - dans une perspective constructiviste. Alors que l'on pourrait envisager qu'un projet urbain ou une opération d'urbanisme sont le produit d'éléments qualifiables d'objectifs (un territoire, des acteurs, une temporalité, un budget, un cadre bâti, etc.), nous défendons ainsi l'idée qu'ils sont d'abord des représentations, c'est-à-dire des constructions individuelles et collectives que les différents acteurs, pour qui ils constituent des enjeux, réalisent et actualisent au gré de leurs expériences.

Nous utilisons ici le terme "représentations» pour qualifier l'ensemble que compose les représentations sociales et individuelles, celles-ci étant comprises comme le "produit et le processus d'une activité mentale par laquelle un individu ou un groupe reconstitue le réel auquel il est confronté et lui attribue une signification spécifique ${ }^{5} »$. Elles permettent aux individus d'appréhender le réel et de choisir comment se comporter. Influencées elles-mêmes par les actions des individus en présence, les représentations sont, ici, des clefs orientant les concepteurs dans toutes les phases du projet dont ils ont la charge ainsi que les échanges qu'ils entretiennent avec les autres acteurs.

Ces représentations, mobilisées dans des temporalités différentes et influencées par des parcours, des valeurs, des références et des manières de percevoir singulières, se construisent par leurs confrontations. En retour, elles participent à «un urbanisme

$2 \quad$ Denise Jodelet, Les représentations sociales, Paris, Presses universitaires de France, 2003 [1989].

3 Gabriel Moser et Karine Weiss, Espaces de vie. Aspects de la relation hommeenvironnement, Paris, Armand Colin, 2003.

4 Hervé Gumuchian, Représentations et Aménagement du Territoire, Paris, Anthropos, 1991. Antoine Bailly et al., Géographie régionale et représentations, Paris, Anthropos, 1995.

5 Jean-Claude Abric, Pratiques sociales et représentations, Paris, Presses universitaires de France, 1987, p. 64. 
concourant ${ }^{6} »$. Le projet en phase de réalisation ou de réception pour les uns, déjà vécu pour les autres, est ici pensé comme une médiation entre deux visions d'un même espace. Par la confrontation de ces deux visions de l'environnement urbain, nous souhaitons comprendre comment elles se construisent et s'influencent mutuellement afin d'appréhender en quoi et surtout pourquoi elles ne se rejoignent pas. Ainsi, espérons-nous montrer comment le sens qu'un acteur, habitant ou concepteur, donne à un territoire dépend à la fois des propriétés de celui-ci, du regard porté sur les autres acteurs, de la manière dont est perçue la production de ce territoire, et de sa propre représentation.

Pour mener à bien cette entreprise, nous nous appuyons sur un double matériau. Le premier, la littérature scientifique, nous permet non seulement de construire notre positionnement théorique mais aussi de poser les enjeux de la production de la ville contemporaine. Il sera l'objet de la première partie de cette contribution. Le second, sur lequel nous fondons notre analyse, est constitué des espaces discursifs collectés lors d'une enquête de terrain menée sur l'écoquartier de Bottière-Chénaie, à Nantes, auprès de ceux qui en font un territoire de projet (techniciens, urbanistes, architectes) et ceux qui en font un territoire reçu et vécu (les habitants) dont nous présenterons quelques éléments dans un second temps. Cette approche dialectique met d'abord en exergue la pluralité de sens qui peuvent être accordés aux propriétés matérielles et symboliques d'un territoire par des individus appartenant à des groupes sociaux distincts. Par l'analyse de quelques confrontations des représentations des acteurs et des habitants, la troisième partie soulignera les relations qui existent entre celles-ci et la nécessité de dépasser la vision simplificatrice d'un processus linéaire du projet : conception, réalisation, réception. L’objectif central de cet article est de démontrer qu'il est nécessaire, dans le champ de recherche, de mettre en avant des modèles qui tentent d'intégrer les notions de

$6 \quad$ François Ascher, Les nouveaux principes de l'urbanisme, La Tour-d'Aigues, Éditions de l'Aube, 2001, p. 98. 
rétroaction ou de récursivité, du moins d'influence des effets sur les causes.

\section{Comprendre la ville en train de se faire}

1.1. La production contemporaine de la ville : enjeux, idéologie et emblèmes

Vouloir saisir les représentations de mêmes espaces pour deux groupes sociaux suggère de comprendre dans quel cadre ces espaces sont produits. En d'autres termes, il s'agit de reconnaître que la pratique urbanistique, et donc les espaces qu'elle fabrique, est guidée par les valeurs de ceux qui les produisent ${ }^{7}$. Par valeurs, nous entendons «ce qui est bien, beau ou juste dans une société donnée ou dans une de ses parties», et elles "apparaissent organisées en système, hiérarchisées à l'intérieur d'un même ensemble social, et variables dans l'espace et le temps, donc socialement construites $^{8} »$. Ainsi, les valeurs précèdent les représentations, auxquelles elles donnent un cadre, une même valeur pouvant se traduire par différentes représentations (la valeur est strictement collective, contrairement à la représentation qui admet des variations interindividuelles ${ }^{9}$ ).

Prétendre analyser et comprendre la production contemporaine de la ville revient à s'interroger sur ce qui la différencie de celles qui l'ont précédée, et plus spécifiquement sur l'emploi systématique des vocables de "projet» et de "développement durable» dans les discours institutionnels, médiatiques et scientifiques (y compris les nôtres) qui paraissent synthétiser les paradigmes centraux de l'urbanisme en ce $\mathrm{XXI}^{\mathrm{e}}$ siècle commençant. Décrypter ces deux termes conduit alors à dévoiler le cœur

$\overline{7}$ Denis Martouzet, Normes et valeurs en aménagement-urbanisme. Limites de la rationalité et nécessité de prise en compte multi-niveaux, Habilitation à diriger des recherches, Bordeaux, Université de Bordeaux 3, 2002.

8 Annie Guédez, Valeurs, dans Jacques Lévy et Michel Lussault (dir.), Dictionnaire de la géographie et de l'espace des sociétés, Paris, Belin, 2003, p. 974.

9 Pascal Moliner, Patrick Rateau et Valérie Choen-Scali, Les représentations sociales. Pratique des études de terrain, Rennes, Presses universitaires de Rennes, 2002. 
des enjeux sociaux, spatiaux et idéologiques de la pratique urbanistique actuelle.

Emblème organisationnel de la modernité occidentale ${ }^{10}$, le projet se définit comme une "procédure stratégique, pragmatique et contextuelle de fabrication intentionnelle de l'urbain qui tend à se substituer à la planification standard - téléologique, théorique et universelle ${ }^{11} »$. Parce que le projet est un vecteur efficace de la production d'objets à la fois idéels et matériels correspondant parfaitement aux grands courants de valeurs des années 1990, qu'il est devenu la figure emblématique de l'urbanisme contemporain. Plus qu'une procédure technique, outil et processus médiatique et organisation, il est un instrument de gouvernance et renvoie aux valeurs de l'idéologie néo-libérale qui l'a fait naître $^{12}$. De la sorte, l'enjeu du développement économique devient central pour le projet et il porte la double contrainte de conformité et de distinction qui s'impose aujourd'hui aux villes et aux agglomérations.

Le projet se trouve, de façon générale, pris dans la double exigence de flexibilité générique et de spécificité. Il en va ainsi du projet urbain qui est censé être celui d'une ville particulière (comme on parle du projet d'une personne) dont il permettrait même d'affirmer l'identité et qui clame simultanément une nécessité universelle d'adaptation au Monde, une veille par rapport aux possibles opportunités de développement ${ }^{13}$.

L'une des «nouveautés» introduite en urbanisme par le projet est alors l'importance de la notion de contexte, ce qui marque une rupture notamment par rapport à la prétention universelle du modernisme. Il s'agit alors pour les concepteurs de le revendiquer à la fois dans leurs réalisations et dans leur communication.

10 Jean-Pierre Boutinet, Anthropologie du projet, Paris, Presses universitaires de France, 2005 [1990].

11 Laurent Devisme, "Projet urbain", dans Jacques Lévy et Michel Lussault (dir.), Dictionnaire de la géographie et de l'espace des sociétés, Paris, Belin, 2003, p. 747.

12 Gilles Pinson, «Le projet urbain comme instrument d'action publique», dans Pierre Lascoumes et Patrick Le Galès (dir.), Gouverner par les instruments, Paris, Presses de la Fondation Nationale des Sciences Politiques, 2004. Luc Boltanski et Ève Chiapello, Le nouvel esprit du capitalisme, Paris, Gallimard, 1999.

13 Laurent Devisme, op. cit. 
Cependant, si le projet urbain rompt avec l'ambition universaliste dans sa mise en œuvre matérielle, son idéologie demeure, elle, universelle : le projet se retrouve partout, à toutes les échelles de décision et de réalisation. En cela, il participe alors ardemment à la diffusion généralisée du mode de gestion managériale, audelà du champ de l'entreprise. Instrument de gouvernement libéral par excellence, le projet introduit un véritable bouleversement idéologique par rapport à la programmation urbaine, changeant l'ordre des priorités ${ }^{14}$.

Ainsi, dans le projet urbain, l'accent n'est [...] plus mis sur les contraintes juridiques opposées aux interventions des acteurs privés, notamment économiques, mais sur la valorisation des atouts, des avantages comparatifs de la ville, tout ce qui peut favoriser l'implantation des entreprises et de leurs cadres ${ }^{15}$.

Le projet va donc servir la recherche d'attractivité des collectivités et le marketing territorial, ni un à-côté du projet ni un aspect secondaire de celui-ci; il est à la fois un aspect central et un objectif. En effet, c'est ce marketing qui conduit les projets urbains à intégrer (pour ne pas dire à être conditionnés par) les nouvelles préoccupations réelles ou supposées des acteurs économiques et les populations cibles dans un contexte de compétitivité entre les territoires.

En d'autres termes, non seulement le projet déplace les enjeux du résultat vers le processus, mais porte également davantage sur l'innovation dans les modes de gestion plutôt que sur les objectifs sociaux et spatiaux à atteindre. Cependant, si le processus évolue alors nettement par rapport à la programmation traditionnelle, le projet n'introduit pas une évolution radicale des représentations et des manières de fonctionner des concepteurs d'espace :

Les changements observés affectent essentiellement la démarche, sans conduire les professionnels de l'aménagement à rompre fondamentalement leur enfermement dans la culture techniciste ou artistique qui fait de l'usage un «angle mort». Leurs pratiques traduisent alors, tantôt une

\footnotetext{
14 François Ascher, op. cit.

15 Gilles Pinson, op. cit., p. 204.
} 
fascination récurrente pour la mode, tantôt des frilosités notamment à travers le fétichisme du patrimoine bâti ${ }^{16}$.

Le projet, portant aussi la marque des représentations spécifiques de ses concepteurs, est alors le processus qui permet d'imposer la conception dominante de la ville contemporaine : celle de la ville comme produit.

Les projets actuels portent le sceau du développement durable, cet « ensemble de représentations qui structure le débat politique contemporain ${ }^{17}$ » qui fournit un cadre de référence pour l'action en visant théoriquement à l'inscription de «la sphère des activités humaines dans un espace-temps élargi, celui de la planète considérée dans son devenir ${ }^{18}$ ». Dans la pratique urbanistique, ce cadre idéologique se manifeste avant tout par le renouvellement du vocabulaire performatif et des concepts qu'il s'agit de mettre en œuvre, à toutes les échelles, selon le célèbre slogan "penser global, agir local» avec de nouveaux mots clefs comme «biodiversité», "changement climatique», "performance énergétique», «mixité sociale» ou «vivre-ensemble».

Définissant l'idéologie comme un "système d'idées, de jugements et de valeurs possédant des capacités organisatrices d'un groupe humain et des pratiques de ses membres ${ }^{19}$ ", nous pouvons lire les actions sur l'espace et l'espace lui-même comme des productions idéologiques, du moins des manifestations de la mise en œuvre d'une idéologie : celle du développement durable. En effet, l'objectif premier d'une idéologie est de désigner les buts légitimes de l'action ${ }^{20}$. En cela, elle est un système de contrôle s'appuyant sur les valeurs et leur hiérarchisation, et donc plus

\footnotetext{
16 Ibid.

17 Cyria Emelianoff, Rémy Kafou et Mathis Stock, «Développement durable», dans Jacques Lévy et Michel Lussault (dir.), Dictionnaire de la géographie et de l'espace des sociétés, Paris, Belin, 2003, p. 249.

18 Ibid.

19 Christian Ruby, «Idéologie», dans Jacques Lévy et Michel Lussault (dir), Dictionnaire de la géographie et de l'espace des sociétés, Paris, Belin, 2003, p. 481.

20 Florence Giust-Desprairies, "Représentation et imaginaire», dans Jacques Barus-Michel, Eugène Enriquez et André Lévy (dir.), Vocabulaire de psychologie. Positions et références, Ramonville Saint-Agne, Erès, 2004, p. 231-250.
} 
largement sur la diffusion de représentations conformes à ces valeurs. Dans le champ de la production de l'urbain, on parlera alors d'idéologie spatiale ${ }^{21}$, considérant qu'elle participe de l'idéologie générale.

Dans le cas présent, l'urbanisme durable peut être considéré comme l'idéologie spatiale du développement durable, lui-même structuré par l'idéologie capitaliste. Nous pouvons alors le considérer comme un référentiel, proposant certes des solutions opérationnelles, mais surtout comme un système hiérarchisé de valeurs et de jugements fournissant un cadre aux représentations mobilisées par l'action politique et urbanistique, sous la condition, bien entendu, de leur correspondance aux valeurs durabilistes.

Renforcés par la circulation de "packages technologiques", communément nommées «bonnes pratiques ${ }^{22}$ ", les projets d'aménagement portant le sceau du durable se traduisent alors par l'application de procédés de conception et de fabrication standardisés, l'emploi d'outils identiques (zones d'aménagement concertées, sociétés d'économie mixte, sociétés publiques locales d'aménagement, etc.) et d'instruments normatifs (chartes, démarches, labels, etc.). En cela, les projets urbains des années 2000 et 2010 ne sont pas moins comparables entre eux que les lotissements pavillonnaires des années 1980-90 ou même encore les grands ensembles des années 1950-60. Ils sont les figures de la production contemporaine de la ville. Chaque période formule ainsi différemment les impératifs qui doivent guider la production de son espace. Si le développement durable met à l'agenda de l'urbanisme contemporain un certain nombre d'enjeux, il s'appuie, en les faisant parfois évoluer, sur des outils, des dispositifs ou des références hérités des reconfigurations progressives précédentes et, notamment, des structures économiques et sociales préexistantes. Nous considérons alors l'urbanisme durable, dans

$\overline{21}$ Anne Gilbert, «L'idéologie spatiale : conceptualisation, mise en forme et portée pour la géographie", L'Espace Géographique, vol. 15, n ${ }^{\circ}$, 1986, p. 57-66. tiques" dans les opérations urbaines, entre normes et fabrique locale», Espaces et sociétés, vol. 4, $\mathrm{n}^{\circ} 131,2007$, p. 15-31. 
sa mise en œuvre quotidienne, comme la norme de la production urbanistique contemporaine. Ainsi, "nouvelle doxa ${ }^{23}$ ", le développement urbain durable est bien l'idéologie dominante de la production urbaine dans de nombreux pays occidentaux.

Nous employons le terme d' $\mathrm{d}^{\text {"idéologie dominante }}{ }^{24}$ » parce que l'urbanisme durable s'impose socialement comme une évidence légitime et parce que sa nature idéologique est alors masquée par la force incontestable de l'évidence, ce qui pousse à la méconnaissance des mécanismes qui l'instituent. Il ne s'agit alors nullement d'en nier les spécificités par rapport aux formes précédentes mais de la repositionner comme telle, et non comme cas exceptionnel ou comme un objectif qu'il conviendrait d'atteindre à tout prix. Reconnaître cela revient aussi à reconnaître qu'en plan large, le développement durable est bien une composante d'une idéologie plus vaste, celle du capitalisme libéral, et qu'en plan plus serré le terme écoquartier n'est désormais employé ni plus ni moins que comme synonyme de ZAC. Ainsi, les écoquartiers sont les territoires emblématiques de la production de la ville contemporaine ${ }^{25}$ et c'est pourquoi ils sont des terrains d'étude idéaux pour tenter de la comprendre. Notre objectif n'étant alors en aucun cas de déterminer si ces territoires sont durables ou non mais de comprendre ce qui se construit sous cette appellation, nous considérons que «le durable» est un système de valeurs performatif. Dès lors, est durable ce qui est annoncé comme tel et ce qui, du point de vue de la recherche est important, est d'analyser la manière dont concepteurs et récepteurs l'interprètent.

23 Bernard Cassaigne, "La ville durable», Projet, vol. 6, n 313, 2009, p. 78-83.

24 Pierre Bourdieu et Luc Boltanski, La production de l'idéologie dominante, Paris, Éditions Raisons d'agir, 2008.

25 Benoît Boutaud, "Quartier durable ou éco-quartier?", Cybergeo : European Journal of Geography, 2009; [en ligne] http:// cybergeo.revues.org/index22583. html (consulté le 11 janvier 2011). 
1.2. De l'espace conçu à l'espace reçu : une approche dialectique des représentations de l'urbain

Le vocable de production de l'espace « exprime l'idée que l'espace géographique, en tant que réalité terrestre et sa représentation psychosociale, ne constitue pas un donné naturel, mais, comme le temps, une réalisation collective des sociétés ${ }^{26}$ ». La production de l'espace ne se réduit pas à sa construction matérielle ni au processus économique de la fabrication de biens mais s'élabore dans une dynamique sociale et idéelle. S'intéresser à la production de l'espace, c'est alors affirmer que ce dernier n'est pas une réalité passive, un donné avec lequel il faut composer, mais bien un produit collectif reflétant les valeurs et les représentations de ceux qui le façonnent. Ce sont ainsi les objets qui composent l'espace. Il n'y a pas de substrat préalable, une sorte d'éther, un contenant dans lequel les objets se positionneraient, et ceux-ci sont définissables par les relations qu'ils entretiennent entre eux, et non par leurs seules positions relatives. De là découle que l'espace est aussi (en complément de son statut de construit) un actant, il peut faire agir. Il facilite, incite, parfois oblige; il a une influence, tant par ce qui le compose que par la structure, l'organisation et l'évolution de cette composition ${ }^{27}$.

L'espace ne peut alors être pensé qu’à travers les rapports sociaux. L'espace a donc cette particularité d'être actif dans sa propre production : quotidiennement construit par les rapports économiques et sociaux il les détermine à nouveau activement. En tant que tel, l'espace traduit alors les enjeux de la société qui le produit et notamment le «mode de production» qui l'engendre. D'où l'utilité de la notion de production de l'espace pour penser la ville en train de se faire.

Même si ce terme est parfois considéré comme daté, référant notamment, de manière implicite, à une analyse marxiste de la

26 Guy Di Méo, "Production de l'espace», dans Jacques Lévy et Michel Lussault (dir.), Dictionnaire de la géographie et de l'espace des sociétés, Paris, Belin, 2003, p. 746 .

27 Michel Lussault, L'homme spatial : la construction sociale de l'espace humain, Paris, Seuil, 2007. 
société aujourd'hui peu en vogue, certains auteurs lui préférant les mots de "construction ${ }^{28}$ ", nous le conservons pour trois raisons. La première est une considération pratique sur la clarté de nos écrits : nous employons le terme de construction pour parler du processus de constitution des représentations des individus et des groupes sociaux, la constitution de la ville en tant qu'espace matériel et idéel par ses acteurs est un processus différent, aussi employons nous pour en parler un terme différent. La seconde raison est davantage liée à la domination actuelle de la ville comme produit conçu et mis en œuvre dans une logique marchande et commerciale : si la ville est produit, il semble logique de travailler sur son processus de production. La troisième raison est liée, elle, au concept même de production : nous pensons ainsi que les travaux de conceptualisation de la production de l'espace menés par Henri Lefebvre offrent un cadre théorique tout à fait adapté à l'analyse de la ville contemporaine. Considérant en cela, à la manière d'un Augustin Berque, que «les sociétés aménagent leur environnement en fonction de l'interprétation qu'elles en font, et réciproquement elles l'interprètent en fonction de l'aménagement qu'elles en fon $\mathrm{t}^{29}{ }$ - autrement dit que l'espace révèle autant la vision du monde de la société qui le produit que l'inverse - nous pensons que la manière dont l'espace est produit et dont cette production est vécue éclaire notre compréhension des représentations des acteurs bien au-delà d'une perspective strictement spatiale.

Parler de production de la ville contemporaine nous amène à scinder, à des fins analytiques, le processus spatial en plusieurs niveaux interreliés et correspondant aux différentes réalités que nous envisageons d'observer. Nous proposons de distinguer trois niveaux incluant des représentations distinctes. Tout d'abord, il y a l'espace conçu, l'espace des concepteurs (urbanistes, architectes, aménageurs, etc.) compris comme celui de leurs représentations. Les représentations qui ont conduit à son élaboration

28 Michel Lussault, op. cit.

29 Augustin Berque, Les raisons du paysage. De la Chine antique aux environnements de synthèse, Paris, Hazan, 1995, p. 15. 
s'imposent aux individus qui vont l'utiliser. Il y a ensuite l'espace réalisé, espace matériel directement tributaire du mode de production au sens pratique du terme. Nous le considérons comme inaccessible en tant que tel, et donc comme un élément de médiation entre le premier et le troisième niveau. Ce dernier est l'espace reçu, autrement dit l'espace appréhendé par les usagers tel qu'ils le décrivent en confrontant leurs propres représentations aux traductions symboliques et matérielles de l'espace conçu. Si l'on considère l'espace comme un produit, c'est alors le stade où celui-ci est consommé. Notre ambition est alors de comprendre comment ce dernier niveau se confronte au premier.

Figure 1

Espaces conçu, réalisé et reçu

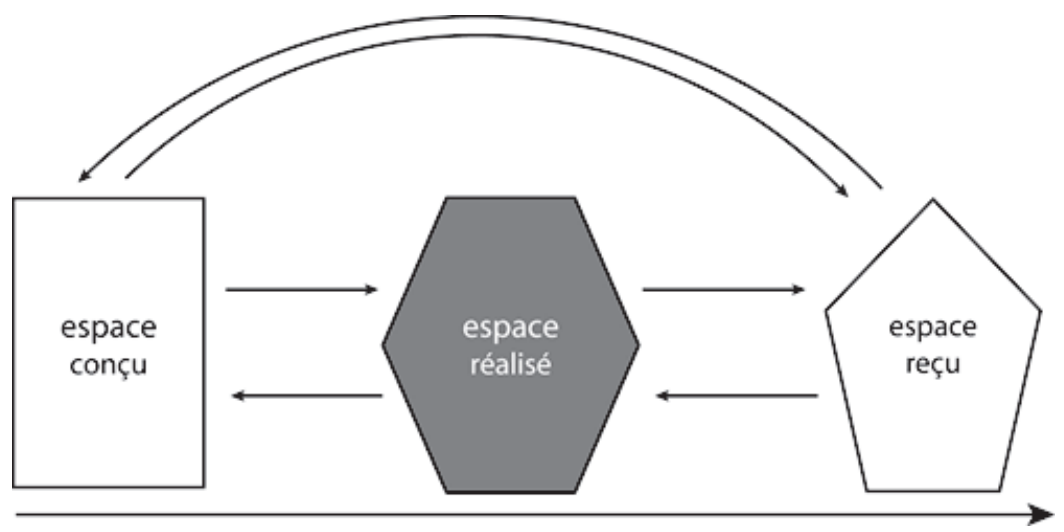

(Conception Matthieu Adam, 2014)

Les représentations permettent aux individus et aux groupes d'appréhender leur environnement afin de "s'y ajuster, s'y conduire, le maîtriser physiquement ou intellectuellement ${ }^{30}$ ». Elles se construisent à partir des expériences, des savoirs, des souvenirs ou encore des valeurs des individus. Notre intérêt pour les représentations vient du fait que, selon nous, elles construisent, et par conséquent révèlent au chercheur, l'idéal, le souhaité, le

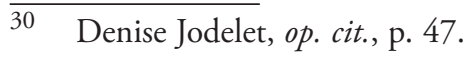


fantasmé ${ }^{31}$, tous trois éléments constitutifs des attentes que les individus expriment vis-à-vis de leurs espaces de vie. Pour appréhender l'espace conçu ainsi comme l'espace reçu, nous devons, d'une part, être en mesure d'accéder aux représentations qui les structurent tous deux et, d'autre part, être capable d'amener des clefs de compréhension sur la manière dont les individus les ont toutes deux construites. En conséquence, le décalage étudié est celui qui existe entre, d'une part, le résultat de l'imbrication des valeurs, références ${ }^{32}$ et interactions qui ont amené les individus à se construire certaines représentations plutôt que d'autres et, d'autre part, ce qui a été mobilisé par les concepteurs pour définir/concevoir ce même espace. Dans cette démarche, le projet urbain est alors envisagé comme un dispositif de médiation entre les représentations des deux groupes sociaux que nous distinguons.

L'hypothèse étant qu'il existe un décalage entre les représentations de ce qu'est un «bon quartier» ou un milieu urbain «de qualité» pour ceux qui le conçoivent et pour ceux qui le reçoivent et que ce décalage est notamment responsable de la déception à la réception, l'espace conçu ne correspondrait pas aux attentes de ses destinataires, et ce, pour un certain nombre de raisons que nous ambitionnons de mettre à jour.

Selon Gustave-Nicolas Fischer :

Il existe un clivage important entre le concepteur et l'usager impliquant deux façons radicalement différentes de considérer l'espace. D’un côté l'espace est plutôt fonctionnel et de l'autre côté il est plutôt vécu c'està-dire investi par une expérience sensori-motrice, tactile, visuelle, affective et sociale... ${ }^{33}$

C'est notamment de ce clivage qu'émergent deux manières différentes de mobiliser des représentations, communes (sociales)

31 Serge Moscovici, «Des représentations collectives aux représentations sociales : éléments pour une histoire", dans Denise Jodelet (dir.), Les représentations sociales, Paris, Presses universitaires de France, 2003 [1989], p. 79-103.

32 Le terme «références» est employé dans cet article pour qualifier des expériences ou des objets qui rendent concrètes et visibles des représentations et participent alors à les formaliser et les accréditer comme "vraies».

33 Gustave-Nicolas Fischer, Psychologie de l'environnement social, $2^{\mathrm{e}}$ édition, Paris, Dunod, 1997, p. 31. 
ou non (individuelles ou liées à des groupes sociaux plus restreints; celles, professionnelles et culturelles des architectes par exemple). Nous posons l'hypothèse que les concepteurs s'appuient sur leurs références, modèles et représentations pour définir un espace synthétisant leur idéal qualitatif et la manière dont ils perçoivent les contraintes et opportunités liées au projet alors que les usagers, eux, jugent l'espace qu'ils perçoivent à l'aune des attentes qu'ils ont pu constituer par rapport à celui-ci suite à leur réception de la commercialisation de l'espace et à partir de l'accumulation de leurs expériences personnelles. Il y a donc bel et bien, outre le clivage décrit par Fischer, un décalage dans les représentations de ce qu'est un «bon» espace ou un espace urbain «de qualité» pour ces deux populations. Précisons que les attentes et projections évoquées ne sont pas figées. En effet, les représentations, à la base de ces mêmes attentes et projections, par essence dynamiques, évoluent au gré de l'évolution du projet et du parcours des individus qui la conçoivent et la reçoivent.

Nous suggérons que ce décalage varie selon les individus et que cette variation est engendrée par un certain nombre de facteurs (individuels, collectifs, spatiaux) influençant pour chacun la construction de l'espace reçu ou conçu. Nous complétons cette hypothèse en ajoutant qu'espace conçu et espace reçu exercent chacun une influence sur l'autre en plus d'être nourris par certaines représentations sociales identiques. Autrement dit, les projections par les concepteurs de l'espace reçu influencent l'espace conçu et les représentations de l'espace conçu (intentions associées) par les usagers influencent l'espace reçu. Nous interrogeons ainsi l'évaluation réciproque de l'espace reçu et de l'espace conçu par les usagers et les concepteurs. 


\section{Confronter les représentations des acteurs à travers leurs discours autour d'un projet urbain emblématique de l'urbain contemporain}

2.1. Le projet urbain comme dispositif de médiation des représentations

Lidée de ce travail est d'aborder les représentations de l'espace et de la ville, car celles-ci sont relativement autonomes du projet qui nous sert de terrain d'étude, de manière générale sans effectuer de hiérarchisation ou de sectorisation a priori. Par exemple, nous n'opérons pas de distinguo entre formes matérielles et organisation sociale de l'urbain, considérant que les champs sont multiples et interdépendants. Les représentations sont pour ceux qui les mobilisent des réductions de la réalité permettant d'analyser cette dernière efficacement et de s'y comporter de manière adéquate. Pour cette raison, il nous semble important d'essayer de ne pas contraindre leur recueil par des réductions supplémentaires apportées par l'observateur ou, du moins, d'être en mesure de les objectiver et de les analyser.

Les représentations étant dynamiques et en perpétuelle évolution $^{34}$, nous pouvons dire qu'elles ont une trajectoire constituée des évolutions successives de la représentation pour un individu et de sa posture vis-à-vis de l'objet de représentation. Cette trajectoire est a priori inconnue même si nous pouvons accéder à certains des éléments qui l'ont influencée ou la conditionnent encore. Que ce soit dans le parcours résidentiel des habitants ou parmi les projets auxquels ils ont participé ou, pour les concepteurs, leur formation, certains éléments constituent des références pour les individus dans la construction de leurs représentations. Ces références viennent nourrir les représentations et influencer leurs trajectoires, tout comme les situations d'interaction, même si celles-ci sont des pistes d'investigation difficilement opération-

$\overline{34}$ Claude Flament, "Structures et dynamiques des représentations sociales», dans Denise Jodelet (dir.), Les représentations sociales, Paris, Presses universitaires de France, 2003 [1989], p. 224-239. 
nelles tant il semble illusoire de prétendre en relever l'historique de manière exhaustive.

Les représentations des concepteurs et des habitants suivent des trajectoires qui sont pour l'essentiel parallèles même si elles peuvent se rejoindre en certains points. C'est notamment le cas lorsqu'un groupe influence suffisamment l'autre pour lui faire partager sa vision du monde. Le projet urbain n'amène pas nécessairement ces trajectoires à se croiser, il peut même dans certains cas les faire dévier dans des directions différentes, en revanche, il marque une référence commune. Cette référence n'unifie pas les trajectoires mais nous offre la possibilité de les observer et de les confronter au travers du temps du projet en tant que séquence d'observation. En raison de notre incapacité pratique à observer ces représentations sur la totalité du projet urbain, de ses prémices à son achèvement, voire sa destruction, cette séquence est en réalité plus contrainte et il serait plus juste de parler d'instant d'observation. Cet instant correspond en l'occurrence à une sorte de mi-temps du projet mobilisé. Mi-temps car il se situe à mi-chemin entre le début et la fin du projet et qu'il permet ainsi de recueillir des paroles au moment où une moitié du quartier est déjà réalisée et habitée mais où celui-ci n'est pas figé, ce qui laisse notamment une part importante à l'activité de projection. Cet instant est à la fois suffisamment tardif pour que les représentations habitantes soient mobilisables et suffisamment hâtif pour que celles des concepteurs ne soient pas altérées par une relecture tardive. En ce qui concerne notre cas d'étude ${ }^{35}$, en réalisant l'enquête en 2012, nous procédions là à une double réduction de la réalité au regard de la temporalité du projet et des caractéristiques des représentations. Toutefois, comme une photographie peut contenir du flou ou du bruit, ces instantanés contiennent un certain nombre d'informations sur l'évolution des représentations de groupes sociaux, de deux ensembles «d'individus interagissant les uns avec les autres et

$\overline{35}$ Nous présentons notre terrain d'étude plus en détail dans le paragraphe 2.2. de cet article. 
placés dans une position commune vis-à-vis d'un objet social ${ }^{36}{ }$. La position commune des habitants vis-à-vis du projet est celle de récepteurs; celle des urbanistes, aménageurs, politiques est celle de concepteurs. Quant aux interactions entre individus, elles sont, dans le cas qui nous préoccupe, au sein du groupe des concepteurs, imposées par le processus du projet urbain, et, dans celui des habitants, garanties, même si cette garantie est minimale, notamment par la coprésence.

Ces deux groupes sociaux, éléments de la société française du début de $\mathrm{XXI}^{\mathrm{e}}$ siècle, baignent dans un ensemble de représentations communes et partagent un certain nombre de valeurs et de références. De plus, ils partagent aussi un certain nombre de représentations préexistantes au projet. D'un côté nous retrouvons celles du groupe des professionnels de la ville, ici essentiellement des architectes et des urbanistes, influencés, entre autres, par leur formation, par le contexte socio-économique d'exercice de leur profession, par les courants de pensée en vogue ou encore par des origines sociales et des orientations idéologiques relativement homogènes. De l'autre, nous avons celles des habitants, groupe socialement homogène pour le projet qui nous intéresse, que l'on peut supposer relativement proches notamment parce qu'elles sont en partie la cause de leur présence.

Dès lors, le projet va avoir une double influence sur ces représentations. Premièrement, par son organisation et les choix qui vont être collectivement retenus lors de la phase de conception, il va faire évoluer les représentations des concepteurs. Interviennent ici la spécificité du projet et l'influence du groupe et de son travail collectif sur les représentations de chacun de ses membres. Le projet va provoquer la mise à l'agenda de certaines thématiques qui seront autant d'objets de représentations, leur formalisation selon certains termes, les renouveler ou les faire évoluer voire, enfin, les conduire à en générer de nouvelles. Si l'on retient l'idée du projet comme construction d'une nouvelle référence qui vient concrétiser la représentation et l'accréditer comme vraie, le travail de conception à la fois individuel et

$\overline{36}$ Pascal Moliner, Patrick Rateau et Valérie Choen-Scali, op.cit., p. 21. 
collectif, en lien avec les autres acteurs du projet, influence directement les représentations individuelles et celles du groupe social en venant les actualiser concrètement avec cette nouvelle référence. Nous avons là une première «boucle de rétroaction" ou de "récursivitée ${ }^{37}$ ".

Deuxièmement, les concepteurs projettent les futurs usagers des lieux et, en se mettant à leur place, cherchent à évaluer leurs représentations. Ils vont donc, par projection ou par retour direct, faire évoluer leurs propres représentations en intégrant ce qu'ils pensent être celles des (futurs) habitants, soit pour s'y adapter, soit pour tenter de les contourner ou de les faire évoluer par l'entremise du projet. Cette évolution des représentations va influencer le projet et venir modifier la nature des interactions qu'ont les concepteurs à la fois entre eux et avec les habitants et les choix techniques et sociotechniques qu'ils vont faire. C'est la seconde boucle de rétroaction.

Figure 2

Le projet urbain comme dispositif de médiation des représentations

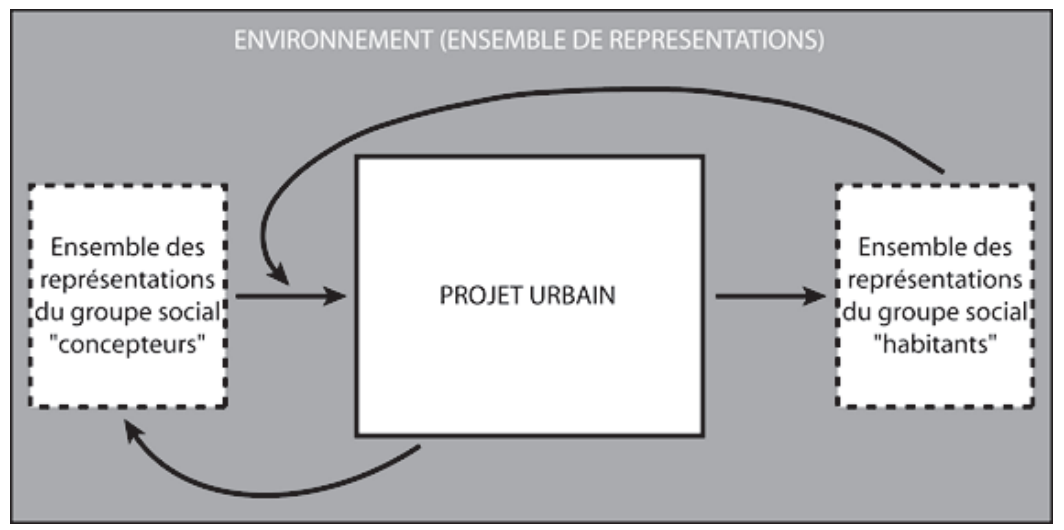

(Conception Matthieu Adam, 2014)

37 Edgar Morin et Jean-Louis Le Moigne, L'intelligence de la complexité, Paris, L'Harmattan, 1999. 
2.2. Saisir les représentations par les discours : implications théoriques et méthodologiques

Pour saisir ces représentations, nous proposons de nous appuyer sur un matériau qui permet de mettre au jour les conceptions individuelles et sociales du réel : des discours collectés dans l'optique spécifique de nos travaux. Il faut ici considérer toute l'importance des discours dans la construction des réalités propres aux deux groupes étudiés. Les discours ne sont pas des objets neutres, ils portent des valeurs et des idéologies et ils sont contextuels, c'est-à-dire situés tant spatialement, temporellement que socialement, signifiant notamment l'appartenance des individus à des groupes sociaux autant qu'ils participent de l'existence même de ces groupes. Si la métaphore de l'espace comme discours a souvent été filée, il s'agit ici de lier discours et espace dans une perspective plus pragmatique que symbolique. Le discours est ici entendu dans son acception la plus simple, à savoir comme le «résultat de l'activité langagière d'un acteur ${ }^{38}$ " produit dans l'action et dans l'interaction. Nous affirmons alors qu'il faut reconnaître l'importance des discours sur l'espace, de tous les discours puisqu'ils sont impliqués dans la construction des espaces de ceux qui les pensent, les dessinent ou les fabriquent et de ceux qui les utilisent plus ou moins quotidiennement.

En effet, le discours [...] ne confère pas uniquement un sens à l'espace, mais en lui donnant des formes, des valeurs, des orientations, des pertinences particulières, il contraint et rend possibles à la fois des pratiques spatiales qui, de façon réflexive, en s'ajustant à lui tel que saisi dans sa configuration discursive, vont l'instaurer et le transformer ${ }^{39}$.

Ainsi discourir sur l'espace est une action à part entière qui ne consiste pas à traduire l'espace matériel en un espace mental communicable en s'appuyant sur des référents extérieurs préexistants et universellement partagés, mais, tout en reflétant les grilles de

$\overline{38}$ Lorenza Mondada, "Discours », dans Jacques Lévy et Michel Lussault (dir.), Dictionnaire de la géographie et de l'espace des sociétés, Paris, Belin, 2003, p. 264. 
lectures et les habitus des individus, à qualifier l'espace et à lui donner du sens.

Les significations que portent le discours se trouvent alors à la fois dans ce qui est dit volontairement (le sens explicite pour le locuteur) et dans ce qui est transmis par les formes elles-mêmes. En effet, ces formes, tout comme le vocabulaire et la syntaxe ${ }^{40}$, ne sont ni vierges ni neutres mais sont aussi porteuses de sens et notamment de valeurs et de représentations ${ }^{41}$. Tout discours est produit dans l'interaction, ce qui signifie qu'il doit être questionné en interrogeant la nature et le contexte de cette interaction. À condition d'être situé dans le temps, l'espace peut être analysé comme un support d'information et décrypté en tant que tel. En résumé, le langage est un matériau adapté pour l'étude des représentations, et des représentations de l'espace en particulier, "parce qu'il est le processus privilégié par lequel les sociétés sémantisent leur environnement et communiquent à son sujet ${ }^{42}$ ».

Parce qu'assigner un vocabulaire aux objets et aux idées permet de qualifier, de regrouper, d'ordonner le réel, l'analyse de discours permet, elle, de faire apparaître les schèmes de perception, d'évaluation et de classification des individus et de les positionner dans l'espace social.

Ainsi, la reproduction d'un ordre social quelconque tolère fort bien de multiples décalages entre les schèmes classificatoires propres aux différentes positions qui s'y inscrivent, mais elle implique aussi que certains de ces schèmes soient l'objet d'un accord suffisamment général pour faire tenir l'ordre dans le registre symbolique ${ }^{43}$.

Dès lors, il s'agit de prendre conscience que «tous les discours structurent la ville selon des régimes d'intelligibilité particu-

$\overline{40}$ La syntaxe est en réalité un révélateur tout aussi pertinent, bien que plus difficilement accessible, particulièrement pour les non-linguistes que nous sommes.

41 Rom Harré, "Grammaire et lexiques, vecteurs des représentations sociales", dans Denise Jodelet (dir.), Les représentations sociales, Paris, Presses universitaires de France, 2003 [1989], p. 149-169.

42 Bernard Debarbieux, «Les problématiques de l'image et de la représentation en géographie», dans Antoine Bailly (dir.), Les concepts de la géographie humaine, Paris, Armand Colin, 2004, p. 205.

43 Jean-Christophe Depaule et Christian Topalov, "La ville à travers ses mots", Enquête, no 4, 1996, non paginé. 
liers ${ }^{44} »$. Le discours d'un locataire de logement social ne structurera pas la ville de la même manière que celui d'un élu, d'un urbaniste, d'un propriétaire ou d'un chercheur. Si les régimes d'intelligibilité sont différents, la portée de tous les discours est, elle aussi, très variable. Ainsi, ils "ne sont pas équivalents en termes de visibilité, de reproductibilité ou d'effectivité sur le terrain. Certaines voix seront reprises, soutenues, commentées, objectivées, amplifiées quand d'autres seront oubliées, étouffées, etcetera ${ }^{45}$ ». Les discours ont un "pouvoir symbolique» et celui-ci dépend grandement de la place des individus qui le portent dans l'espace social ${ }^{46}$. Cette perspective souligne tout l'intérêt qu'il y a à confronter, dans le cadre de nos travaux, des discours venant d'individus dans des postures différentes vis-à-vis d'un même objet.

Inférer des représentations à partir d'un matériau composé de discours recueillis dans le cadre d'entretiens semi-directifs ou de visites de sites, comme c'est le cas dans notre travail, suppose de faire nôtres trois hypothèses méthodologiques fortes. La première est «la capacité de l'acteur à communiquer ses représentations qui ne sont pas toujours aisées à verbaliser ${ }^{47}$ ", la deuxième est la capacité de "l'enquêteur à les comprendre", enfin la troisième est évidemment la pertinence de la méthode de recueil pour «les faire émerger ». Ainsi, prendre comme premier matériau les discours suppose de postuler que les acteurs interrogés, qu'ils soient concepteurs du projet ou habitants, sont compétents pour les produire. Dans ce cas, «la compétence désigne la reconnaissance de l'aptitude de l'individu à la fois à énoncer verbalement l'espace, à le représenter graphiquement, à y exercer des actions, bref à le produire ${ }^{48} \%$. Considérer que les acteurs sont compétents ne

44 Lorenza Mondada, Décrire la ville : la construction des savoirs urbains dans l'interaction et dans le texte, Paris, Anthropos, 2000, p. 39.

45 Ibid.

46 Pierre Bourdieu, Langage et pouvoir symbolique, Paris, Seuil, 2001.

47 Philippe Bongrand et Pascale Laborier, «L'entretien dans l'analyse des politiques publiques : un impensé méthodologique?", Revue française de science politique, vol. 55, nº 1, 2005, p. 98.

48 Marion Ségaud, Anthropologie de l'espace. Habiter, fonder, distribuer, transformer, Paris, Armand Colin, 2012, p. 40. 
signifie pas qu'ils le soient réellement. Ainsi, la compétence est "partagée mais elle ne signifie pas la même chose selon qu'elle appartient au concepteur ou à l'usager ${ }^{49}$ ». Les professionnels ont été formés à manier avec aisance les outils et les mots qui permettent de lire, de projeter et produire l'espace. Ils ne sont cependant pas les seuls à en être capables, et s'ils ne font pas preuve de la même technicité, les habitants sont aussi des acteurs compétents, à même de lire et de juger leurs espaces vie et surtout d'effectuer "le va-et-vient entre le social et le spatial, puisque l'habitant puise les fondements de sa compétence dans les schémas culturels à sa disposition ${ }^{50}$ ». Il en va d'ailleurs de même pour les concepteurs, leur schéma culturel étant simplement pour partie différent.

Conférer cette compétence aux groupes sociaux que nous étudions nous conduit à affirmer que nous pouvons alors saisir au moins une partie de leurs schémas culturels en les interrogeant dans le contexte spécifique de notre enquête. Ainsi, les discours mobilisés sont issus d'une enquête dont la méthodologie a été conçue spécifiquement pour les besoins de cette étude. Elle consiste à rencontrer les habitants puis les concepteurs du projet de Bottière-Chénaie. Les habitants, eux, sont confrontés physiquement au projet réalisé lors de visites libres ${ }^{51}$, ce qui n'est pas le cas des professionnels. En effet, en provoquant la perception par la marche, nous forçons la mobilisation de représentations à la fois pour décider de la visite qui nous sera librement proposée par les habitants (contenu, trajet, durée) et pour produire un discours, sur les lieux traversés ou sur d'autres sujets connexes. De la sorte, les habitants nous livrent la définition vécue de ce qu'ils considèrent comme leur quartier et des éléments de description et de jugement de celui-ci (de ses caractéristiques architecturales à la vie sociale qui s'y installe progressivement en

\footnotetext{
49 Ibid.

50 Ibid., p. 41.

51 Pour une présentation détaillée de la méthodologie employée, lire Matthieu Adam, «Visiter ou faire visiter? La marche comme révélateur du rapport des habitants à leur quartier. L'exemple de visites libres dans l'écoquartier de Bottière-Chénaie», Recherche Transports Sécurité, n 30, 2014, p. 173-189.
} 
passant par la manière dont ils jugent le travail des concepteurs). Ces visites, où nous tenons le rôle d'auditeur bienveillant, n'intervenant que par de rares relances, sont, en complément, pour les enquêtés l'occasion de nous livrer leurs représentations de la ville, leurs rapports à l'espace environnant, leurs «visions du monde».

Cette méthode a notamment pour intérêt de permettre un effacement maximal de l'enquêteur et d'éviter de trop orienter les discours puisque aucune question n'est posée et que tous les propos découlent des choix de l'enquêté de l'interaction qui se produit avec l'espace.

Ceci permet de faire émerger à la fois les enjeux identifiés comme centraux et les représentations qu'ils impliquent. Nous complétons la visite par un entretien semi-directif immédiatement consécutif lors duquel les habitants sont invités à nous conter leur parcours résidentiel et à revenir sur un certain nombre d'éléments ayant trait à leur appréhension et leurs pratiques du quartier. Cette phase de rencontre avec les habitants nous permet d'établir de premiers constats et d'avoir une idée relativement précise de leurs représentations du quartier, du projet urbain mais aussi de ses concepteurs et de leur action. La rencontre avec les concepteurs vient alors dans un second temps. Elle prend la forme, elle, d'un entretien semi-directif plus classique où nous les faisons réagir sur les représentations des habitants et leur demandons de nous parler librement de leur travail, des opérations dont ils avaient la charge, du projet dans son entier, et de leur conception de la production de la ville. Le principe méthodologique pourrait être qualifié de rotatif puisque cela permet des évaluations réciproques ainsi qu'une mise en relation indirecte des différents acteurs. En complément, cette spécificité permet d'unifier au maximum les temporalités spécifiques du projet, de ses acteurs et de l'observateur. 
216 NPSS, VOLUME 10, NUMÉRO 1, 2014

Figure 3

Tracé d'une visite à Bottière-Chénaie

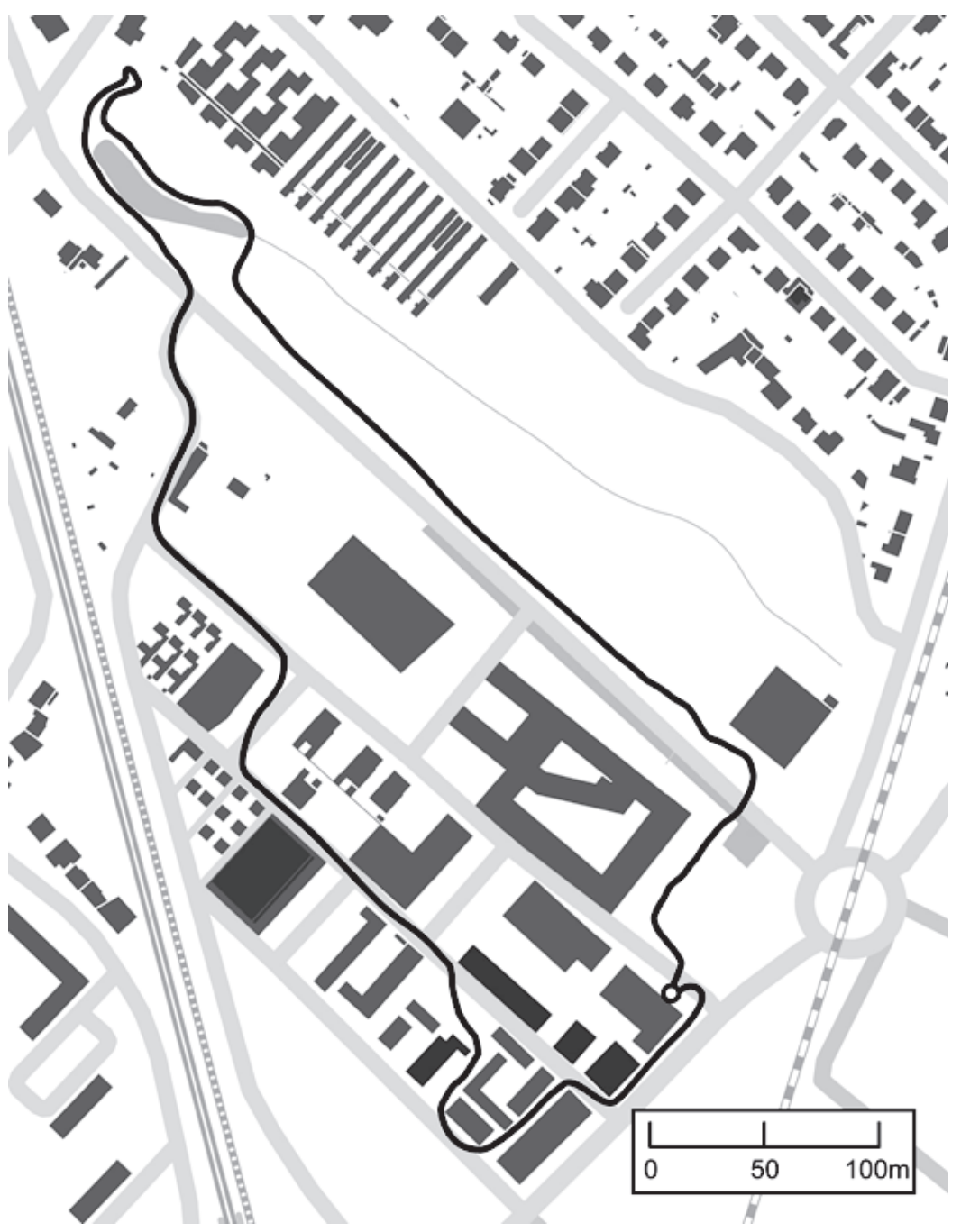

(cartographie de Matthieu Adam, 2013 - fond de carte OpenStreetMap) 
Ce matériau repose alors sur une conception de l'entretien comme évènement interactionnel qu'il est nécessaire de préciser. À l'opposé d'une conception «représentationaliste ${ }^{52}$ » qui conçoit le discours comme "un véhicule neutre et transparent dont la fonction est la transmission des informations", conception qui «implique le plus souvent une intentionnalité communicative du locuteur et le partage d'un code comme condition du bon fonctionnement du discours ", nous préférons donc une conception interactionnelle et praxéologique. Dans cette conception, les objets de discours sont considérés comme n'étant pas donnés à l'avance mais faisant l'objet d'une co-construction impliquant l'enquêté, l'enquêteur, et, dans notre cas, le terrain matériel d'étude : la conception interactionnelle prend alors le discours comme un processus dynamique. Cette approche est intrinsèquement liée à la posture de recherche proposée : si l'espace est considéré comme un objet dynamique que les différents acteurs reconfigurent en permanence, alors les représentations de l'espace sont un processus dynamique de construction du réel, il est donc nécessaire de chercher à les cerner grâce à une méthode s'ajustant dynamiquement à leur contexte d'énonciation.

Nous avons déployé notre méthodologie sur l'écoquartier Bottière-Chénaie, à Nantes ${ }^{53}$. Les habitants enquêtés sont des usagers quotidiens du quartier et les visites ont été réalisées avec des personnes logeant (16 visites) ou travaillant (5) sur le quartier, certaines partageant ces deux aspects (3 visites). Nous avons

\footnotetext{
52 Lorenza Mondada, "L'entretien comme évènement interactionnel», dans Michèle Grosjean et Jean-Paul Thibaud (dir.), L'espace urbain en méthodes, Marseille, Éditions Parenthèses, 2001, p. 198.

53 Ce projet, porté par Nantes Métropole et l'aménageur Nantes Aménagement, est récipiendaire du prix «densité et formes urbaines» du concours EcoQuartier 2009. La réalisation du projet s'accompagne d'une importante communication particulièrement axée sur les thématiques du développement durable. Essentiellement dévolu à l'habitat, avec un objectif d'environ 3000 logements construits à l'horizon 2016, l'écoquartier est richement doté en équipements (bibliothèque, école, gymnase, maison de quartier), en espaces publics et incorpore aussi quelques commerces regroupés autour d'un axe central. Sis entre un quartier pavillonnaire (Chénaie) et un quartier de grands ensembles (Bottière), le quartier neuf (la ZAC) qui s'étend sur 31 hectares est habité depuis 2008.
} 
ensuite rencontré 12 concepteurs du projet, parmi lesquels l'architecte en chef de la ZAC, le paysagiste, le responsable à l'urbanisme de Nantes Métropole, le chargé de mission pour l'aménageur, 7 architectes sur des opérations de logement et d'équipement ainsi que le chargé d'opération d'un promoteur. Les extraits qui suivent sont issus de ces rencontres ${ }^{54}$.

\section{3. Étayer par l'exemple la nécessaire intégration de notions de complexité en recherche urbaine}

3.1. Adhésion, rejets et stratégies de négociation avec l'espace

Le développement durable, qui se présente pour les concepteurs comme une injonction à intégrer un certain nombre de principes et à user d'outils dédiés, est essentiellement perçu par les habitants comme un discours descendant, diffusant ces mêmes principes. Si le développement durable est un enjeu pour ces deux groupes sociaux et dans la mesure où sa définition demeure assez floue, il génère de nombreuses représentations leur permettant de l'interpréter, de s'en saisir, de se l'approprier. Les discours à ce propos révèlent non seulement les représentations, les valeurs et les références que ces deux groupes sociaux mobilisent pour s'y adapter, les rapports qu'ils entretiennent l'un et l'autre, mais aussi la manière dont ils se perçoivent mutuellement. Ainsi, ces mêmes discours montrent de quelles manières les rapports à l'espace s'inscrivent dans une confrontation à la fois à celui-ci, aux discours sur celui-ci et à ceux qui les portent.

Même si les acteurs s'accordent à défendre les valeurs consensuelles que sont l'intégration des enjeux environnementaux et sociaux dans le production de l'urbain, il y a chez eux un scepticisme général vis-à-vis de l'injonction qui leur est faite de concevoir leurs projets dans un cadre perçu comme de l'affichage ne renouvelant finalement qu'assez peu la structure de la production de la ville qui, elle, reste essentiellement dictée par des contraintes économiques. Le développement durable est souvent

54 Le format d'anonymisation que nous avons choisi doit se lire ainsi : C pour concepteur, $\mathrm{H}$ pour habitant, $\mathrm{N}$ pour Nantes [une enquête du même type a été menée à Lyon (projet Confluence)], le numéro associé est aléatoire. 
appréhendé comme n'étant qu'un concept à la mode et comme une somme d'éléments de discours qu'il convient d'intégrer aux projets :

Je pense que c'est beaucoup d'affichage. Chaque période a son truc comme ça où un moment $\mathrm{y}$ a des lieux qu'on peut aménager et on les labélise en disant "voilà c'est un écoquartier" et les maires arrivent avec ça, c'est politiquement correct. Mais si en fouillant on avait trouvé du pétrole, c'est sûr que là on aurait pas fait un écoquartier, on aurait pris la nappe de pétrole et c'aurait fait de l'économie quoi $(\mathrm{CN} 1)$.

D'autres pointent ainsi le fait que n'importe quel projet puisse s'appeler écoquartier ou quartier durable et qu'en conséquent, cela ne signifie pas (plus) grand-chose. Dans ce contexte, l'affichage du développement durable serait avant tout une nécessité pour vendre les projets.

Quand le ministère a lancé cet appel à projet on a considéré qu'on était dans le sujet [...] Sachant qu'il y a toujours une envie, une sorte d'envie de faire valoir, et Nantes est experte pour ça. [...] C'était un des éléments importants du dossier Nantes capitale verte européenne 2013. Donc c'est un objectif politique d'affichage, je crois que c'est ça essentiellement. [...] très vite quand quelque chose marche on l'insère dans toute cette politique d'attractivité (CN4).

Puis, il y a, chez certains architectes comme chez certains urbanistes, une méfiance affichée vis-à-vis de l'efficacité autoproclamée. Par exemple, les panneaux solaires, la ventilation double flux, etc. seront de la "quincaillerie écologique». Plus profondément, s'exprime une certaine lassitude à l'endroit d'un discours qui se répète sans cesse et qui, à force, apparaît consensuel et lisse. Certains dénoncent le dessin d'une ville propre, nette, sans accroc, responsable, mais "sans saveur».

En ce moment ça m'énerve ces histoires d'écoquartier. Je n'en peux plus de cette idée d'une ville confortable, saine. C'est une idée de la ville où tout est beau, propre, rangé, aimable. La ville c'est pas ça! Une ville c'est un lieu d'expression, un lieu de contact, d'action. [...]. Et je le supporte pas parce que c'est un vocabulaire qui est une espèce de poudre aux yeux dans l'idée d'une ville qui serait confortable (CN8).

Tous considèrent que le durable ne devrait pas être l'objectif majeur et tous parlent de qualité, urbaine, environnementale ou 
architecturale, ne précisant que très peu ce que signifie alors le vocable de qualité. Cette critique récurrente apparaît ainsi comme une tentative de distanciation ou de déresponsabilisation vis-à-vis de l'espace produit mais aussi des discours officiels autour de celui-ci. Ainsi, si parmi les concepteurs aucun ne croît réellement au développement durable (même si par ailleurs tous adhèrent à l'essentiel des valeurs qu'il prône) alors pourquoi tous s'en réclament-ils? Et, deuxièmement, si ce n'est que de l'affichage, qui en est responsable puisque tous communiquent sur le sujet et participent ainsi à la diffusion généralisée d'un même message?

Sans répondre ici directement à ces deux questions, mentionnons que la même appréciation du développement durable se retrouve dans les discours de nombre d'habitants. L'idée d'affichage, d'effet de mode, apparaît lorsque certains des enquêtés disent ouvertement qu'il s'agit de communication ou de marketing ou, lorsque d'autres nous interrogent sur la raison nous conduisant à leur parler d'écoquartier. Davantage qu'une critique du marketing urbain, les habitants décrivent une distorsion entre l'affichage d'un certain nombre d'ambitions, notamment environnementales, et une réalité nettement moins aboutie ou, justement, ambitieuse. Ainsi, nombreux sont ceux qui doutent que le quartier soit aussi durable que le préfixe «éco" le laisse entendre ou qui font référence explicitement ou implicitement à l'idée de greenwashing.

Ouais, qui est un peu superfétatoire comme on dit! [rires] Je trouve que c'est un mot qui va bien avec ce qu'on en fait. C'est-à-dire que c'est très vitrine... Donc voilà c'est cette disparité là, c'est ce que j'appelle moi la vitrine... [...] c'est la marge qui fait la différence entre le projet initial et le résultat quoi. C'est-à-dire qu'à un moment on oublie les objectifs humains et on se concentre sur des objectifs qui sont plus politiques et c'est ça qui est regrettable (HN11).

Afin de ne pas alourdir le propos, nous ne détaillerons pas les aspects sur lesquels porte l'idée d'un décalage entre l'écoquartier tel qu'il devrait être et tel qu'il se présente. En revanche, analysons les deux étapes de ce processus de décalage. 
Les deux représentations qui se confrontent ne sont pas exactement celle de la réalité matérielle des lieux d'un côté et celle de ce qui est montré ou vendu de l'autre. Il s'agit plus de la confrontation de la représentation de ce que devrait être le quartier s'il correspondait à l'idée d'écoquartier proposée par la communication officielle et de ce qu'il est, au final, matériellement. Difficile de parler de publicité mensongère, dans la mesure où les discours officiels autour du projet n'ont jamais essayé de vendre un écrin de verdure, ni prétendu qu'il n'y aurait que des technologies de chauffage alternatives ou des bâtiments techniquement très performants. En revanche, en utilisant le terme d'écoquartier, ces producteurs de discours renvoient directement à cette représentation et expriment des attentes que le projet ne comble pas. Nous constatons alors que le développement durable urbain, du moins la notion d'écoquartier, jouit déjà d'une représentation sociale. Utiliser ce terme, revient donc à sousentendre qu'on va réaliser un projet qui doit correspondre à cette représentation. Alors, quoi de plus légitime, lorsque ce n'est pas le cas, que le fait que les habitants perçoivent un décalage?

De manière simplifiée, cette représentation est celle des écoquartiers d'Europe du Nord, Bedzed ou Vauban au premier chef. Dans les entretiens réalisés, ces références reviennent très souvent pour désigner, a contrario de Bottière-Chénaie, ce qui serait un «vrai» écoquartier.

Après faut pas se voiler la face, je pense que les gens comprennent les choses, on sait bien qu'on est pas forcément... Le terme écoquartier, certes c'est écoquartier mais pour avoir justement voyagé à l'extérieur je sais bien qu'il y a un côté complètement comm'. C'est pas la vision d'un écoquartier comme on pourrait avoir (HN17).

Cette représentation exprime une certaine esthétique qui serait celle du développement durable urbain : une végétation conséquente, l'utilisation du bois, des toitures végétalisées, etc. Cela semble l'expression d'une adhésion, chez nombre d'habitants, aux «valeurs» que porte le développement durable.

À l'opposé de cette posture d'adhésion on retrouve logiquement celle du rejet. Chez un certain nombre d'habitants, nous 
observons une remise en cause d'un discours perçu comme moralisateur et normatif ou comme de la poudre aux yeux. En un sens, il y a une saturation de discours qui se traduit par des propos extrêmement critiques à l'encontre d'un certain nombre d'éléments assimilés comme étant le fruit de ce discours. C'est aussi l'expression d'une lassitude par rapport à celui-ci et conséquemment la revendication de pratiques ou de valeurs contraires : " oui je circule toujours en voiture», "non je ne trie pas tous mes déchets", "non je ne veux pas réduire ma consommation de papier en refusant la publicité». C'est la revendication du fait de ne pas être "un bon élève».

Pour autant, rejeter le discours officiel ne signifie pas que l'on se sent mal dans le quartier ou que l'on regrette d'être venu y habiter. Il faut clairement faire le distinguo entre le rapport entretenu avec l'espace en tant que tel et avec le discours qui s'y rapporte. Autrement dit, certains disent aimer leur quartier, le trouver agréable à vivre et en être satisfaits pour une multitude de raisons diverses tout en rejetant fortement le récit officiel qui en est fait. En un sens, nous pouvons même avancer que ceux qui ne placent aucune attente dans les ambitions en termes de développement durable sont plus élogieux vis-à-vis de leurs lieux de vie puisqu'ils ne les analysent et ne les évaluent pas à l'aune de ce qu'ils devraient être mais en fonction de ce qu'ils en retirent, et ce, sans se préoccuper de la correspondance avec les valeurs affichées.

Ce que nous enseigne cette opposition entre le jugement porté par le discours sur l'espace et l'espace lui-même, c'est que les habitants sont rarement et totalement dans une posture ou une autre. Pour l'essentiel, ils «font avec» les propriétés matérielles et symboliques de ces espaces, en un mot négocient avec l'espace et ses valeurs. En prenant l'exemple de l'idée que le quartier ne serait pas aussi durable que ce que la communication veut bien en dire, la négociation à laquelle se livrent les habitants va consister à tenter de faire se rapprocher l'image du quartier tel qu'il devrait être et le quartier tel qu'il est perçu. Cette posture poursuit l'objectif de réduire le décalage entre ces deux représentations 
pour augmenter la satisfaction résidentielle. Alors, nous pouvons distinguer deux types de stratégies qui illustrent de quelles manières la réception de l'espace (pratiqué, vécu) est inséparable de l'accueil réservé au discours sur l'espace (de projets) ainsi qu’à ceux qui le portent.

La première est une stratégie individuelle qui consiste à prendre ses distances vis à vis du discours, à le mettre à l'écart, pour n'évoquer le quartier que comme un lieu de vie, et ce, sans se soucier du fait qu'il soit présenté comme durable. Les habitants qui adoptent cette stratégie vont donc agir sur leur prise en compte du discours officiel pour altérer le décalage entre celui-ci et l'espace tel qu'il est perçu. Nous récoltons alors des propos qui consistent à dire que même si cela ne va pas aussi loin que cela pourrait, les ambitions affichées amènent quand même à ce que l'attention prêtée à l'aménagement du quartier soit supérieure à celle que l'on pourrait trouver dans des quartiers moins exposés. Les habitants vont alors défendre l'idée selon laquelle cet affichage rassure sur la qualité du projet ou le respect des derniers standards ou normes en vigueur, en un mot que le projet est moderne et bien mené.

Est-ce que ça change quelque chose maintenant? Non, juste dans le sens où ça me rassure, où je me dis qu'il va pas y avoir n'importe quoi de fait. Voilà, c'est juste ça... mais après ça change rien du tout (HN10).

C'est la représentation des ambitions affichées qui sera ici réduite.

Quant à la seconde, elle est totalement inverse et consiste à se servir du discours officiel et de celui des concepteurs pour agir ou tenter d'agir sur l'espace. Plus qu'un rejet de l'espace tel qu'il est, va alors s'opérer la construction d'une opposition à ceux qui sont considérés responsables de ce décalage perçu entre communication et réalité : la municipalité, l'aménageur, les promoteurs, etc. Cette stratégie va, contrairement à la première, être collective et se construire contre ceux qui ont la responsabilité de ce qui a été fait. Responsabilité de l'aménagement de l'espace d'une part, responsabilité du contenu des discours officiels d'autre part. On retrouve ici l'opposition classique entre gouvernants et gouvernés. 
La stratégie des habitants va alors consister à tenter de se servir du contenu du discours officiel pour faire pression sur le contenu du projet.

Mais faut se battre, faut se battre contre eux en plus, alors qu'ils ont déjà une logique d'écoquartier donc ils devraient plus nous aider qu'autre chose. [...] Je reste sur l'idée que c'est un écoquartier... et même si c'en est pas vraiment un, je ferais tout pour que ça le devienne. Donc pour moi, j'ai déjà inclus l'idée que c'était un écoquartier, c'est déjà un écoquartier... à nous de faire en sorte que nos actions puissent étayer le fait qu'on appelle ça un écoquartier. Et je pense qu'on peut être aidé par la ville et tout ça... (HN14)

L'idée centrale est qu'une fois l'ambition de faire du développement durable affichée et communiquée, elle engage les responsables, notamment les responsables politiques. Ces derniers seraient alors en obligation de s'y tenir, faute de quoi ils perdraient toute légitimité à se réclamer du développement durable. L'enjeu du rapport de force pour les habitants va donc être de mettre politiques et techniciens face à ce qui est présenté comme une incohérence pour les enjoindre à la faire disparaître ou, du moins, à l'estomper. Des habitants de Bottière-Chénaie ont ainsi mobilisé cette stratégie pour obtenir la réalisation d'un composteur collectif, d'autres envisageaient au moment de notre enquête de se servir du fait que Nantes était capitale verte européenne en 2013 pour faire avancer leurs revendications. L'objectif est ici de rapprocher l'espace tel qu'il est perçu de la représentation de ce qu'il devrait être.

3.2. Des regards qui se croisent : l'exemple de la mixité sociale

Des principes qui s'imposent à la ville contemporaine dans le contexte de la généralisation du développement durable, on ne retient souvent que la mise à l'agenda des enjeux environnementaux. Mais, quand bien même les projets urbains actuels, et particulièrement les écoquartiers, sont avant tout conçus pour les classes moyennes et supérieures et inaccessibles aux plus fragiles socialement et économiquement ${ }^{55}$, le récit de la ville durable

55 Jacques Theys et Cyria Emelianoff, "Les contradictions de la ville durable», Le Débat, no 113, 2001, p. 122-135. 
insiste sur l'importance de réaliser des projets consacrant une certaine mixité sociale. Ce vocabulaire n'est pas neutre mais comme de nombreux autres concepts performatifs de l'urbanisme contemporain, ses contours n'apparaissent pas d'une netteté absolue. Cela conduit à la construction de multiples représentations. Cet exemple met particulièrement en exergue la mobilisation de références dans la construction des représentations mais surtout la manière dont celles des deux groupes sociaux que nous étudions s'imbriquent, se superposent et finalement s'influencent mutuellement.

Dans le projet de Bottière-Chénaie, une analyse pragmatique en termes de mélange de populations fait apparaître une mixité en réalité très réduite. Entre les locataires de logements sociaux relativement onéreux et une population de primo-accédants s'inscrivant pour beaucoup dans des politiques locales ou nationales d'aide à l'accession, l'écoquartier est socialement homogène. Cependant, le politique a fait de la mixité sociale un axe fort de communication et le présente comme un enjeu central de sa politique, en écho aux considérations nationales ${ }^{56}$. Ce vocabulaire renvoie à un environnement de représentations assez large, comprenant des valeurs, comme le mélange, la solidarité, l'égalité, différentes représentations de la culture ou du multiculturalisme, de la pauvreté, des classes sociales, de la différences, de la hiérarchisation, etc. et un certain nombre de références essentiellement négatives, celles de la non-mixité, comme les grands ensembles ou les gated communities. Cette question trouve sa place dans les débats actuels sur l'intégration, l'égalité des chances et le développement durable. La mixité est alors un enjeu pour les deux groupes sociaux étudiés, pour les concepteurs car elle est une injonction généralisée formulée par les pouvoirs publics, pour les habitants parce qu'elle impacte directement la composition sociale de leurs lieux de vie. Ils ont donc besoin de s'appuyer sur un certain nombre de représentations pour savoir à quoi s'en

56 Yves Sintomer "Mixité sociale et lutte pour l'égalité», Mouvements, vol. 3, $\mathrm{n}^{\text {os }} 15-16,2001$, p. 218-220. Dominique Schnapper, «Idéal et limites de la mixité sociale», Informations Sociales, n ${ }^{\circ} 125,2005$, p. 6-15. 
tenir et pour prendre position. Loin d'être palpable, la mixité sociale est ainsi l'exemple type du phénomène de représentations puisque ce sont elles, et uniquement elles, qui viennent donner une réalité à ce concept.

Le concept de mixité sociale suscite une forte adhésion chez les architectes et les urbanistes. Ne remettant pas en question le mode de faire l'urbain, il condense les principes des deux modèles de ville qui leur servent de repoussoirs absolus : l'habitat pavillonnaire et les grands ensembles. Au premier, est reproché sa faible qualité paysagère, l'espace qu'il consomme, son homogénéité sociale et l'individualisme qu'il mettrait en avant en plaçant notamment l'automobile au cœur du mode de vie de ses habitants. Au second, est reproché son universalisme et notamment l'aspect générique et déconnecté des particularités locales de ses formes urbaines et surtout ce que sont socialement devenus les grands ensembles, perçus comme des "ghettos». La mixité sociale se présente alors comme une tentative de ne pas reproduire les erreurs du passé en ciblant l'enjeu du mélange de population. Ainsi, une partie de l'adhésion des urbanistes et des architectes au projet de la ville durable tient au fait qu'elle se présente comme un retournement des deux figures repoussoirs. Cette croyance en un "vivre ensemble» renouvelé, et aujourd'hui à nouveau du domaine du possible grâce au projet et à la ville durables, se fonde sur le fait que la proximité spatiale entraînerait mécaniquement la mixité sociale et que la fréquentation de classes sociales différentes en un même espace permettrait de tirer tout le monde vers le haut. C'est notamment une vision stéréotypée de la classe populaire qui aurait besoin des classes supérieures pour mieux vivre. Ce qui fait référence ici, ce sont les grands ensembles et l'urbanisme moderne qui sont des figures répulsives, ce qu'il faudrait absolument éviter.

Mais si on se contraint pas à faire de la mixité, qu'on fait des quartiers en disant "voilà, là on va mettre tous les HLM", on a vu ce que ça a donné, c'est parfait, ça fait des ghettos [...] La mixité, je pense que ça permet aussi à tout le monde, aussi bien aux gens qu'on catalogue comme étant logement sociaux d'être tirés un peu vers le haut en voyant comment fonctionnent les autres, mais aussi aux gens qui habitent à 
côté de se dire que les logements sociaux c'est pas forcément que ce que je décrivais de façon un petit peu trash tout à l'heure $(\mathrm{CN} 8)$.

Plus généralement, on se place ici à l'échelle des valeurs : le mélange de population apparaît ainsi comme une valeur qui s'auto-justifie sans besoin d'argumentation, ce qui est d'ailleurs une des caractéristiques des valeurs ${ }^{57}$. Ainsi, si les enquêtés expriment d'une manière générale des doutes vis-à-vis des discours politiques sur le sujet, les représentations qu'ils ont de la non-mixité les poussent à une adhésion forte à la valeur qui les sous-tend. Ainsi, leur demander de justifier pourquoi l'idée de mixité était une bonne chose nous a conduit à nous heurter à des discours d'incompréhension, comme si l'adhésion, si ce n'est au vocable de mixité, du moins à l'idée de mélange de populations, allait de soi, nous faisant clairement sentir que ne serait-ce qu'exprimer un doute serait rompre avec une certaine vision progressiste (celle du mélange) et dévier clairement de ce qui est acceptable. Que les discours politiques sur la mixité sociale puissent cacher des politiques de gentrification dans certains cas ${ }^{58}$ ou que le remplacement progressif des populations d'extraction populaire de certains quartiers par des personnes issues de la petite bourgeoisie (ou des classes moyennes pour emprunter un vocabulaire plus lisse) puisse aussi avoir des conséquences négatives est une idée qui semble très éloignée de leurs considérations. Le projet vient formaliser cette valeur à laquelle les concepteurs adhèrent sous un certain angle et notamment avec certains termes, la mixité sociale correspond à certaines traductions spatiales et à certains outils qui influencent directement les concepts qui lui sont associés. Concrètement, cela se traduit par une répartition de logements sociaux sur le quartier, par un affichage des valeurs qu'est censée prôner la mixité et, in fine, par la réalisation de ces logements et le côtoiement qu' elle induit. La manière dont elle se met concrètement en place vient alors influencer la vision qu'en ont les concepteurs. Apparaissent alors

57 Denis Martouzet, op. cit.

58 Jean-Pierre Garnier, Une violence éminemment contemporaine. Essais sur la ville, la petite-bourgeoisie intellectuelle et l'effacement des classes populaires, Paris, Agone, 2010. 
des doutes dans les discours tenus par les concepteurs. De plus, si leur adhésion à l'idée de mélange, en tant que valeur, est totale, en revanche celle aux manières dont les élus se réclament et mettent en œuvre la mixité sociale est vertement critiquée. Cette distanciation s'explique d'une part par le qualificatif de "poudre aux yeux" donné aux discours officiels - le développement durable en tête - et d'autre part par une vive critique à l'encontre de la législation loi SRU. Toutefois, au-delà, demeure l'idée que les modalités actuelles de mixité sont "mieux que rien» et que cela va dans la direction qu'il convient de suivre.

Les habitants se réfèrent plus ou moins aux mêmes valeurs mais réagissent différemment. Nous constatons à la fois une adhésion d'un certain nombre de personnes à ces dites valeurs et la génération d'une représentation de la mixité comme une richesse palpable sur le quartier. Néanmoins, nous observons aussi un certain scepticisme ou un rejet du concept, associé à l'idée de quotas, qui apparaissent enfermant, ainsi qu'à la mobilisation de représentations, aisément qualifiées de clichés, sur les pauvres et leurs mœurs, sur l'immigration, la délinquance, les profiteurs.

Si leur usage est loin d'être systématique, en revanche, ces représentations sont fortement présentes dans l'anticipation ou la projection de la réception. Pour le dire vite, les architectes et les urbanistes voient les futurs habitants comme des êtres individualistes faisant passer leur confort avant l'intérêt général et adoptant une posture de rejet de la valeur mélange et, à l'inverse, d'adhésion à celle de l'entre-soi. De fait, ils projettent la mixité sociale comme source probable de frictions et adaptent alors le projet pour tenter de limiter le conflit. On retrouve ici la vision négative des habitants perçus comme nécessairement réticents à l'idée de mixité et incapables de comprendre la nécessité du mélange. D'où selon eux la nécessité de communiquer sur le sujet et de réaliser des logements qui soient le plus diffus, les plus intégrés possible au cadre bâti du quartier, voire de véritablement éduquer les habitants des logements sociaux pour que les frictions soient les plus faibles possibles. Cette communication et le travail 
réalisé sur l'intégration des logements sociaux viennent alors influencer les représentations des habitants. Ce qui illustre alors comment les représentations des habitants et des concepteurs s'influencent en permanence de façon indirecte.

Figure 4

Trajectoires des représentations de la mixité sociale

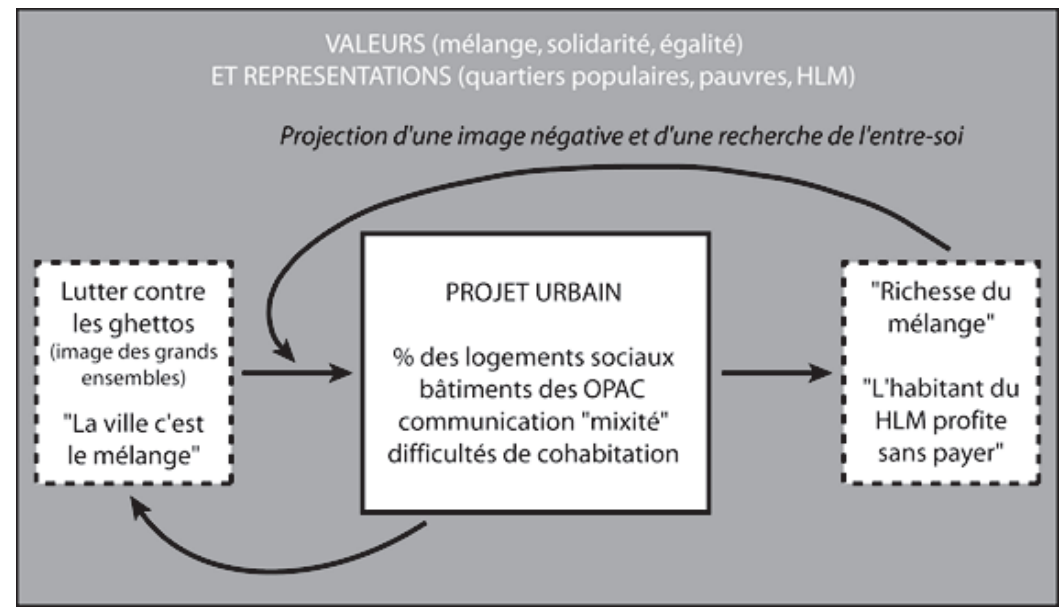

(Conception Matthieu Adam, 2014)

L'exemple de la mixité sociale éclaire le découpage que nous effectuons entre espace conçu, espace réalisé et espace reçu. Ainsi, les actions sur l'espace matériel, qu'elles soient aussi fortes que sa conception et sa réalisation ou d'ampleur plus faible comme les multiples agencements à la marge que réalisent les usagers, intègrent les représentations des acteurs. Elles sont motivées par des valeurs, des idéologies, des représentations symboliques attribuées à l'espace par ceux qui agissent sur lui. Par exemple, on retrouve dans le choix de l'emplacement, des fonctions ou de l'architecture d'un bâtiment de logement social des déterminants symboliques plus ou moins conscients. Autant qu'il est vecteur de représentations, l'espace matériel est enjeu de représentations ${ }^{59}$. Symétriquement, les représentations mentales sont fortement

59 Muriel Rosemberg, Le marketing urbain en question : production d'espace et discours dans quatre projets de villes, Paris, Economica Anthropos, 2000. 
conditionnées par l'espace matériel : les formes, les matériaux, les climats, les ambiances, les temporalités, la composition sociale influencent le rapport que l'on entretient avec un lieu et les représentations qu'on s'en fait. L'approche constructiviste consiste alors à constater qu'il est illusoire de prétendre accéder directement à l'espace matériel et qu'il faut alors se concentrer sur la compréhension de son appréhension, autrement dit sur l'espace mental. Parallèlement, si, pour des raisons analytiques ou méthodologiques, on distingue l'espace représenté de ceux qui représentent, adopter une approche constructiviste revient à considérer l'espace matériel comme un média/support de réalités différentes qu'il influence et qui l'influencent. Admettre que l'on ne peut avoir accès à l'espace qu'à travers les représentations qu'en ont différents individus revient à accepter qu'on ne peut penser l'espace que dans l'interaction et que séparer une éventuelle réalité du monde physique des réalités des individus qui l'usent est une entreprise illusoire. Pour reprendre les mots de Martin Heidegger, cela revient à considérer que l'homme et l'espace ne forment qu'un : "nous parlons de l'homme et de l'espace, ce qui sonne comme si l'homme se trouvait d'un côté et l'espace de l'autre ${ }^{60} »$. Par conséquent, cette posture revient à accepter le jeu de la "double herméneutique " qu'Anthony Giddens attribue aux sciences sociales : ce que nous observons et interprétons est une réalité que les acteurs que nous étudions ont déjà transformée par l'observation et l'interprétation ${ }^{61}$.

Dans la perspective qui est la nôtre, on peut alors considérer que l'idée centrale que les représentations spatiales médiatisent [la] relation entre l'espace physique et l'individu afin que ce dernier puisse s'approprier l'espace comme un espace d'actions. Pour résumer, ni les caractéristiques des individus, ni celles du milieu physique, ne peuvent expliquer séparément les représentations spatiales car c'est à la jonction de ces deux entités qu’elles sont générées ${ }^{62}$.

$60 \quad$ Martin Heidegger, Essais et conférences, Paris, Gallimard, 1980, p. 186.

61 Jean Nizet, La sociologie de Anthony Giddens, Paris, La Découverte, 2007.

62 Thierry Ramadier, "Les représentations cognitives de l'espace : modèles, méthodes et utilité», dans Gabriel Moser et Karine Weiss (dir.), Espaces de vie. Aspects de la relation homme-environnement, Paris, Armand Colin, 2003, p. 178. 
En ce qui nous concerne, nous ajoutons à ce constat le fait que ces représentations sont aussi d'une part le fruit des interactions plus ou moins directes entres groupes sociaux et entres membres de ces groupes sociaux et d'autre part des perceptions qu'ils ont d'eux-mêmes et des autres. Ainsi, leur considération dans une perspective d'étude visant à comprendre avec finesse et justesse les rapports au territoire, malgré la difficulté à les appréhender, ne doit pas être négligée.

\section{Conclusion}

Entrelacs de propriétés matérielles et symboliques mais aussi de jeux et de représentations de leurs acteurs, les territoires sont indéniablement des objets complexes. Dans le champ de l'analyse et de la compréhension de la production de la ville contemporaine, les premiers enseignements portés ici au débat vont dans le sens qu'il y a une pertinence à associer l'étude des représentations à la notion de complexité. Le projet, à la fois mode de production de la ville contemporaine (au sens opérationnel du terme) et traduction in situ de l'idéologie dominante (le développement durable), est bien plus que la somme des représentations de ceux qui le conçoivent et de ceux qui le reçoivent. Dès lors, il est difficile de penser tant la conception que la réception d'un projet urbain sans considérer que représentations, valeurs et références soient collectives et/ou individuelles et s'entrelacent en permanence. Cela revient à acter que scinder le processus de construction d'un territoire en étapes, du type conception, réalisation, réception, est trop limitatif.

La validation de nos hypothèses tout comme la consolidation de notre modèle sont à poursuivre. D'une part, cela passerait par l'analyse de ce qui, dans la définition même donnée au territoire, diffère entre un savoir expert (celui des chercheurs), un savoir à visée opérationnelle (celui des experts) et un savoir usager (celui des habitants - dont certains appartiennent en même temps aux autres catégories). D'autre part, l'exploration minutieuse de comment, pourquoi et à quel moment ces définitions s'écartent ou se rejoignent en serait le second volet. Dès lors, pensons-nous, 
s'ouvre la porte à véritable opérationnalité du modèle que nous proposons et à la mise en œuvre d'une méthodologie d'analyse capable d'être mobilisée dans le cadre d'une étude urbaine.

\section{Bibliographie}

Adam, Matthieu, «Visiter ou faire visiter? La marche comme révélateur du rapport des habitants à leur quartier. L'exemple de visites libres dans l'écoquartier de Bottière-Chénaie», Recherche Transports Sécurité, n 30 , 2014, p. 173-189.

Abric, Jean-Claude, Pratiques sociales et représentations, Paris, Presses universitaires de France, 1987.

Ascher, François, Les nouveaux principes de l'urbanisme, La Tour-d'Aigues, Éditions de l'Aube, 2001.

Bailly, Antoine et al., Géographie régionale et représentations, Paris, Anthropos, 1995.

Berque, Augustin, Les raisons du paysage. De la Chine antique aux environnements de synthèse, Paris, Hazan, 1995.

Boltanski, Luc et Ève Chiapello, Le nouvel esprit du capitalisme, Paris, Gallimard, 1999.

Bongrand, Philippe et Pascale Laborier, «L'entretien dans l'analyse des politiques publiques : un impensé méthodologique?", Revue française de science politique, vol. 55, $\mathrm{n}^{\circ}$ 1, 2005, p. 73-111.

Bourdieu, Pierre, Langage et pouvoir symbolique, Paris, Seuil, 2001.

Bourdieu, Pierre et Luc Boltanski, La production de lidéologie dominante, Paris, Éditions Raisons d'agir, 2008.

Boutaud, Benoît, "Quartier durable ou éco-quartier?", Cybergeo : European Journal of Geography, 2009; [en ligne] http:// cybergeo.revues.org/ index22583.html (consulté le 11 janvier 2011).

Boutinet, Jean-Pierre, Anthropologie du projet, Paris, Presses universitaires de France, 2005.

Cassaigne, Bernard, «La ville durable», Projet, vol. 6, $\mathrm{n}^{\circ}$ 313, 2009, p. 78-83.

Choay, Françoise, La règle et le modèle : sur la théorie de l'architecture et de l'urbanisme, Paris, Seuil, 1996 [1980]. 
Debarbieux, Bernard, «Les problématiques de l'image et de la représentation en géographie», dans Antoine Bailly (dir.), Les concepts de la géographie humaine, Paris, Armand Colin, 2004, p. 199-211.

Depaule, Jean-Christophe et Christian Topalov, «La ville à travers ses mots", Enquête, n 4, 1996, p. 247-266.

Devisme, Laurent, «Projet urbain», dans Jacques Lévy et Michel Lussault (dir.), Dictionnaire de la géographie et de l'espace des sociétés, Paris, Belin, 2003, p. 747-748.

Devisme, Laurent, "Urbanisme», dans Jacques Lévy et Michel Lussault (dir.), Dictionnaire de la géographie et de l'espace des sociétés, Paris, Belin, 2003, p. 962-966.

Devisme, Laurent, Marc Dumont et Élise Roy, «Le jeu des «bonnes pratiques" dans les opérations urbaines, entre normes et fabrique locale», Espaces et sociétés, vol. 4, no 131, 2007, p. 15-31.

Di Méo, Guy, «Production de l'espace», dans Jacques Lévy et Michel Lussault (dir.), Dictionnaire de la géographie et de l'espace des sociétés, Paris, Belin, 2003, p. 746-747.

Emelianoff, Cyria, Rémy Kafou et Mathis Stock, «Développement durable», dans Jacques Lévy et Michel Lussault (dir.), Dictionnaire de la géographie et de l'espace des sociétés, Paris, Belin, 2003, p. 249-251.

Fischer, Gustave-Nicolas, Psychologie de l'environnement social, $2^{\mathrm{e}}$ édition. Paris, Dunod, 1997.

Flament, Claude, «Structures et dynamiques des représentations sociales», dans Denise Jodelet (dir.), Les représentations sociales, Paris, Presses universitaires de France, 2003 [1989], p. 224-239.

Garnier, Jean-Pierre, Une violence éminemment contemporaine. Essais sur la ville, la petite-bourgeoisie intellectuelle et l'effacement des classes populaires, Paris, Agone, 2010.

Gilbert, Anne, "L'idéologie spatiale : conceptualisation, mise en forme et portée pour la géographie ", L'Espace Géographique, vol. 15, nº 1, 1986, p. 57-66.

Giust-Desprairies, Florence, «Représentation et imaginaire», dans Jacques Barus-Michel, Enriquez, Eugène et André Lévy (dir.), Vocabulaire de psychologie. Positions et références, Ramonville Saint-Agne, Erès, 2004, p. 231-250.

Guédez, Annie, "Valeurs», dans Jacques Lévy et Michel Lussault (dir.), Dictionnaire de la géographie et de l'espace des sociétés, Paris, Belin, 2003, p. 974-975.

Gumuchian, Hervé, Représentations et Aménagement du Territoire, Paris, Anthropos, 1991. 
Heidegger, Martin, Essais et conférences, Paris, Gallimard, 1980.

Harré, Rom, "Grammaire et lexiques, vecteurs des représentations sociales», dans Denise Jodelet (dir.), Les représentations sociales, Paris, Presses universitaires de France, 2003 [1989], p. 149-169.

Jodelet, Denise, Les représentations sociales, Paris, Presses universitaires de France, 2003 [1989].

Lefebvre, Henri, 2000, La production de l'espace, Paris, Anthropos, 2000 [1974].

Lussault, Michel, L'homme spatial: la construction sociale de l'espace humain, Paris, Seuil, 2007.

Martouzet, Denis, Normes et valeurs en aménagement-urbanisme. Limites de la rationalité et nécessité de prise en compte multi-niveaux, Habilitation à diriger des recherches, Bordeaux, Université de Bordeaux 3, 2002.

Moliner, Pascal, Patrick Rateau et Valérie Choen-Scali, Les représentations sociales. Pratique des études de terrain, Rennes, Presses universitaires de Rennes, 2002.

Mondada, Lorenza, Décrire la ville : la construction des savoirs urbains dans l'interaction et dans le texte, Paris, Anthropos, 2000.

Mondada, Lorenza, "L'entretien comme évènement interactionnel», dans Michèle Grosjean et Jean-Paul Thibaud (dir.), L'espace urbain en méthodes, Marseille, Éditions Parenthèses, 2001, p. 197-214.

Mondada, Lorenza, "Discours", dans Jacques Lévy et Michel Lussault (dir.), Dictionnaire de la géographie et de l'espace des sociétés, Paris, Belin, 2003, p. 264-265.

Morin, Edgar et Jean-Louis Le Moigne, L'intelligence de la complexité, Paris, L'Harmattan, 1999.

Moscovici, Serge, «Des représentations collectives aux représentations sociales : éléments pour une histoire», dans Denise Jodelet (dir.), Les représentations sociales, Paris, Presses universitaires de France, 2003 [1989], p. 79-103.

Moser, Gabriel et Karine Weiss, Espaces de vie. Aspects de la relation hommeenvironnement, Paris, Armand Colin, 2003.

Nizet, Jean, La sociologie de Anthony Giddens, Paris, La Découverte, 2007.

Pinson, Gilles, "Le projet urbain comme instrument d'action publique", dans Pierre Lascoumes et Patrick Le Galès (dir.), Gouverner par les instruments, Paris, Presses de la Fondation Nationale des Sciences Politiques, 2004, p. 199-233.

Ramadier, Thierry, «Les représentations cognitives de l'espace : modèles, méthodes et utilité», dans Gabriel Moser et Karine Weiss (dir.), Espaces 
de vie. Aspects de la relation homme-environnement, Paris, Armand Colin, 2003, p. 177-200.

Rosemberg, Muriel, Le marketing urbain en question : production d'espace et discours dans quatre projets de villes, Paris, Economica Anthropos, 2000.

Ruby, Christian, «Idéologie», dans Jacques Lévy et Michel Lussault (dir), Dictionnaire de la géographie et de l'espace des sociétés, Paris, Belin, 2003, p. 481-482.

Schnapper, Dominique, "Idéal et limites de la mixité sociale», Informations Sociales, $\mathrm{n}^{\circ} 125,2005$, p. 6-15.

Ségaud, Marion, Anthropologie de l'espace. Habiter, fonder, distribuer, transformer, Paris, Armand Colin, 2012.

Semmoud, Nora, La réception sociale de l'urbanisme, Paris, L'Harmattan, 2007.

Sintomer, Yves, «Mixité sociale et lutte pour l'égalité», Mouvements, vol. 3, $\mathrm{n}^{\text {os }} 15-16,2001$, p. 218-220.

Theys, Jacques et Cyria Emelianoff, «Les contradictions de la ville durable», Le Débat, n 113, 2001, p. 122-135. 